\title{
Love addiction: Reply to Jenkins and Levy
}

\author{
Brian D. Earp, Bennett Foddy, Olga A. Wudarczyk, \& Julian Savulescu
}

We thank C. S. I. Jenkins and Neil Levy for their thoughtful comments on our article about love and addiction (Earp, Wudarczyk, Foddy, \& Savulescu, in press). While we do not have the space for a comprehensive reply, we would like to touch on a few main issues.

Jenkins points out, correctly in our view, that the word 'addiction' can trigger "connotations of reduced autonomy." It may therefore be used, she argues, to (illegitimately) “excuse" violent or otherwise harmful actions-disproportionately carried out by men, she stresses - within the context of romantic relationships. Debates about love addiction, therefore, "are best addressed with an eye to the more general issue of how we as a society apportion responsibility for things like date rape and intimate partner violence" (Jenkins, in press, p. x).

Jenkins is right to call attention to this fraught backdrop, and we agree that the word 'addiction' must be applied carefully. The last thing we would want to do is to create an "excuse" for violent behavior, if that meant taking such behavior less seriously, whether from a socio-ethical or legal perspective. Therefore, we should be extremely skeptical about easy appeals to addiction, especially by way of self-diagnosis, insofar as the goal is to downplay one's culpability for causing harm. This just goes to show how important it will be to solve the underlying "mystery" of autonomy and responsibility as it relates to genuine love addiction (as we highlighted in our paper).

In solving this mystery, we must of course be on the look-out for mere excusemaking. But based on the evidence we reviewed, we should also be open to the possibility that some forms of romantic attachment really could entail a loss of control in certain circumstances. This loss of control—especially if it became associated with violent behavior - might reasonably influence our judgments about ultimate moral responsibility (depending upon a range of factors), but it would by no means necessarily justify a more "lenient" response. In fact, if it could be shown that a 
person "really couldn't help" his or her out-of-control, harmful behavior-on account of a bona fide romantic addiction - then forcible intervention of some kind could possibly be justified, up to and including involuntary confinement. At the end of the day, understanding the true etiology of harmful behavior, including in cases in which diminished autonomy is indeed a contributing factor, will be necessary for designing effective strategies for preventing it.

Jenkins also worries about our use of the word 'love.' She cites the work of another author, Bell Hooks, who argues that "genuine" love is incompatible with the sorts of toxic relationship characteristics we used to illustrate romantic addiction. On a practical level, she writes that "using the word 'love' - with all its attendant positive connotations and associations - to describe [harmful] relationships can be a dangerously rhetorically effective way of concealing how bad they really are" (p. x).

We agree that such rhetoric can be problematic. Indeed, given this type of concern, we are sympathetic to the normative argument that the word 'love' should only be used to describe relationships, feelings, attitudes, forms of romantic attachmentetc. - that are conducive to the flourishing of everyone involved. In other words (according to this argument), if it isn't positive, happy, or healthy, it isn't 'really' love. We have no disagreement with those who choose to use 'love' in this restricted way, nor would we want to go out of our way to defend an alternative definition. We would simply note that, throughout history, and in much of Western literature, romantic love has been variously described as a sickness, a form of insanity, and even a threat to the social order - calling attention to the power of romantic passion to interfere with our higher-order desires, goals, commitments, and obligations (see Earp, Wudarczyk, Sandberg, \& Savulescu, 2013). This use of the term, which has clearly negative connotations, remains common enough that we felt it was appropriate given the aims of our essay.

Turning now to Levy. His commentary focuses on the term 'addiction,' which he suggests we have 'hijacked' from the literature on drug addiction-much as so-called drugs of abuse are said to 'hijack' the mesolimbic dopamine system — and misapplied it to the case of love. Now, Levy seems to acknowledge the psychiatric consensus (given in the DSM-V) that gambling can lead to a genuine state of addiction. Yet gambling clearly does not dysregulate the mesolimbic dopamine system in the way 
that some addictive drugs do, by direct manipulation of the dopamine receptors or stores. The fact that gambling is so clearly addictive poses a problem, we suggest, for 'hijacking' accounts of addiction.

Levy sketches a solution to this problem in his reply: gambling maintains an ability to provoke the dopaminergic learning signal by providing unexpected rewards, so every gambling win must be processed as a signal "that the world is better than expected" (p. x). Since the gambler can never reliably predict that her gambling behavior will result in a reward, her brain will never down-regulate the dopamine signal when she wins. As a result, every win will continue to reinforce the gambling behavior-unlike, we might imagine, the food reward that the monkeys in Schultz's experiment received (Schultz, Dayan \& Montague 1997).

On its face this seems like a neat solution to the problem that gambling addictions pose for the hijacking view, but it doesn't quite work.

"Addictive love" maintains an ability to provoke the dopaminergic learning signal by providing unexpected rewards, so every "romantic event" must be processed as a signal "that the world is better than expected". This particularly true of pathological lovers, such as psychopaths and people with borderline personality disorder, who randomize their love rewards. Abusive relationships have just this pattern of random rewards.

This seems a very accurate description of just the problem with pathological love.

In addition, it isn't clear that the mechanism of problem gambling depends on the intermittent reward schedule, even though randomness is an archetypal property of games used for gambling. Many of the forms of gambling that frequently lead to addiction (lotteries, for example) pay out so seldom that it cannot possibly be the intermittent monetary payout that provides the neural reward. Even for gambling with a classically intermittent payout schedule (gambling machines, for example) neuroimaging studies suggest that addicted gamblers stop responding to wins as reward states, and begin to show a reward signal upon seeing the simulated spinning of the machine's wheels — a reward that is utterly reliable (Shao et al. 2013). 
But second, even if Levy's explanation of gambling addiction were correct, it doesn't seem like that would weigh against the conception of love as an addiction. If gambling 'hijacks' the reward system by providing unpredictable rewards, that would mean that any unpredictable reward would have the power to 'hijack' the reward learning systems. This, in turn, would suggest that the category of 'hijacking rewards' is rather a lot larger than just drugs and gambling, since behaviors with unpredictable rewards are extremely common. Indeed, the rewards associated with romantic love and sex are particularly unreliable, given that they depend so greatly on the responses of another human being, and in fact some of the most harmful outcomes in romantic love are known to be reinforced by intermittent or unreliable expressions of affection (Miller, Lund \& Weatherly 2012; Meloy 1996).

In short, if any unpredictably rewarding behavior can 'hijack' the reward learning system, including behaviors that have existed throughout most of our evolutionary history, then even Levy's preferred account of addiction - the 'hijacking' accountcannot rule the love-related phenomena we discuss in our paper.

\section{References}

Earp, B. D., Wudarczyk, O. A., Foddy, B., \& Sevulescu, J. (in press). Addicted to love: What is love addiction and when should it be treated? Philosophy, Psychiatry, \& Psychology, in press.

Earp, B. D., Wudarczyk, O. A., Sandberg, A., \& Savulescu, J. (2013). If I could just stop loving you: Anti-love biotechnology and the ethics of a chemical breakup. American Journal of Bioethics, 13(11), 3-17.

Jenkins, C. S. I. (in press). 'Addicted'? to 'Love'?. Philosophy, Psychiatry, \& Psychology, in press.

Levy, N. (in press). Hijacking addiction. Philosophy, Psychiatry, and Psychology, in press.

Meloy, J.R. (1996). Stalking (obsessional following): A review of some preliminary studies. Aggression and Violent Behavior, 1: 147-162. 
Miller, K.B., Lund, E., and Weatherly, J. (2012) Applying Operant Learning to the Stay-Leave Decision in Domestic Violence. Behavior and Social Issues 21:135-151

Shao, R., Wakeley, J., Behrens, T., and Rogers, R. (2013). Shifts in reinforcement signalling while playing slot-machines as a function of prior experience and impulsivity. Translational Psychiatry, 3, e213

Schultz, W., Dayan, P. and P.R. Montague. 1997. A neural substrate of prediction and reward. Science 275: 1593-159. 